\title{
Evaluación de la Calidad de un Tramo de Río Urbano Intervenido y Selección de un Paso para Peces, Región del Biobío, Chile
}

\author{
Alejandro I. Morales ${ }^{(1)}$ y Catterina P. Sobenes ${ }^{(1,2) *}$ \\ (1) Departamento de Medio Ambiente y Energía, Facultad de Ingeniería, Universidad Católica de la \\ Santísima Concepción. Alonso de Ribera 2850, Concepción, Chile (e-mail: aimorales@ing.ucsc.cl) \\ (2) Centro de Investigación en Biodiversidad y Ambientes Sustentables, Universidad Católica de la \\ Santísima Concepción. Alonso de Ribera 2850, Concepción, Chile (e-mail: csobenes@ucsc.cl)
}

* Autor a quien debe ser dirigida la correspondencia

Recibido Ago. 5, 2017; Aceptado Oct. 10, 2017; Versión final Nov. 3, 2017, Publicado Abr. 2018

\begin{abstract}
Resumen
En el presente estudio se evaluó la calidad de un tramo del sistema fluvial urbano Estero Nonguén en la región del Biobío de Chile. Este estero ha sido encauzado en su rivera, con el objetivo de evitar futuros desbordes y afectar a las poblaciones aledañas a éste. Sin embargo, no han sido evaluadas las intervenciones al sistema fluvial para verificar y evitar que se altere el desplazamiento natural de la ictiofauna presente. Para esto, se analizó la presencia de barreras en un tramo del estero mediante indicadores de calidad y conectividad. Para recomendar una alternativa de paso de peces en un sitio identificado con una barrera, se utilizó el método de ponderación lineal. El tramo del río analizado resultó ser de calidad moderada y la conectividad deficiente, lo que implicaría que existirían grupos de peces nativos que no se pueden desplazar aguas arriba ni aguas abajo de la barrera. Para restablecer la conectividad del sistema fluvial, se propone construir como paso un dispositivo de rampa de piedras, que es un sistema de bajo costo, de tránsito sencillo y acorde al ambiente ribereño.
\end{abstract}

\section{Quality Evaluation of a Disturbed Urban River and Fish Pass Selection, Biobío Region, Chile}

\begin{abstract}
In this study the quality of a section of the fluvial system known as Estero Nonguén located in the Biobío region in Chile was evaluated. This river was canalized for avoiding future floods and affecting surroundings towns. However, there have been no conducted evaluations on interventions to the river system to check and avoid damage to the natural movements of fish in the river. The presence of barriers in a section of the river was analyzed with quality and connectivity index. To propose a fish pass for a section of the river with an obstruction, the lineal ponderation method was used. The quality of the river was moderate with a deficient connectivity, blocking the movement of a group of fish over the obstruction. To reconnect the river, it is proposed the construction of a stone ramp, because of its low cost and simple flow, in compliance with the river environment.
\end{abstract}

Keywords: urban river fragmentation; fish pass; conservation; migration; urban rivers 


\section{INTRODUCCIÓN}

La fragmentación de los distintos ecosistemas naturales provocada por las actividades humanas, es uno de los mayores factores que contribuyen a la reducción de la biodiversidad y abundancia de especies (Vince, 2010; Gardner, 2011). Este problema es particularmente importante en los ecosistemas ribereños, donde las represas, desviadores de agua, alcantarillas, tuberías y otras estructuras civiles crean barreras para el movimiento natural de los organismos acuáticos (Washington Department of Fish and Wildlife, 2009; Kemp, 2012). Este tipo de obras pueden provocar diferentes efectos como: reducción de hábitat, pérdida de vegetación acuática o despeje de vegetación ribereña (Martínez, 2001; Swales 1989). La fragmentación de los ríos provoca la pérdida de biodiversidad, y por lo tanto altera el funcionamiento natural de los ecosistemas (Morita y Yamamoto, 2002; Morita y Yokota, 2002; Williams et al., 2005; Goodwin et al. 2006; Mims y Olden, 2012). Por ello, la restauración del "river continuum" (Vannote et al., 1980), o continuidad del río, constituye uno de los mayores desafíos para la conservación biológica, gestión de los recursos naturales y de la ingeniería. Si es que existen obstáculos en un sistema ribereño y la fauna íctica no puede desplazarse aguas arriba o aguas abajo del obstáculo, es probable que se produzca una declinación en las poblaciones de peces (Brooker, 1985), alterando el estado ambiental del ecosistema ribereño (Jackson et al., 2000).

Para mitigar el impacto de las obras civiles sobre la migración natural en los peces, desplazamiento natural que puede ser hacia aguas arriba o aguas abajo del río, se utilizan en países desarrollados los dispositivos de paso para peces, los que consisten en infraestructuras hidráulicas cuya finalidad es permitir el paso de los peces sorteando el obstáculo del curso fluvial (Pena et al., 2006). Existen distintos diseños: pasos rústicos, pasos de estanques sucesivos, esclusas para peces, ascensores para peces, entre otros (Elvira et al., 1998). Los pasos naturalizados se adaptan mejor para obstáculos de pequeña y mediana altura (Sanz-Ronda et al. 2011) existiendo los pasos rústicos, rampa de piedra y el río artificial (Sanz-Ronda et al., 2011, 2013; Trapote, 2009). Sin embargo, la mayoría de los pasos han sido diseñados para salmónidos migratorios, lo que no ha favorecido en la abundancia de especies nativas de menores tallas (Mallen - Cooper y Brand, 2007).

En Chile la ictiofauna presenta un alto endemismo, relacionado directamente con el nivel de aislamiento que presentan las cuencas hidrográficas chilenas comparado con las demás cuencas de Sudamérica (Habit y Victoriano, 2012). Son de tamaño pequeño (Vila et al., 1999) con tallas $<15 \mathrm{~cm}$ de longitud total en su estado de adultos, y las más grandes sólo alcanzan tallas máximas de $40 \mathrm{~cm}$ (Vila y Habit, 2015), lo que implica que debido a su tamaño les resulta difícil sortear obstáculos. A pesar de la validez internacional de los pasos de peces (Kemp, 2012), en gran parte de los países latinoamericanos no han sido implementados (Pompeu et al., 2012). Los desplazamientos aguas arriba de las especies nativas continentales chilenas son fundamentales para los procesos reproductivos y mantención de las poblaciones naturales (Piedra et al., 2012). Considerando que en Chile no existen pasos de peces, el presente estudio buscó evaluar la calidad de un tramo de un sistema ribereño urbano que se encuentra encausado y proponer un paso de peces para el sistema ribereño, como una primera etapa necesaria para solucionar la interrupción de las migraciones aguas arriba y aguas debajo de la Ictiofauna nativa.

\section{MATERIALES Y MÉTODOS}

A continuación, se describe el área de estudio, y a continuación los indicadores utilizados. Por último, se indica la forma de selección de un paso de peces a proponer en cada caso. Esta metodología considera métodos implementados en Europa.

\section{Área de Estudio}

El estudio se realizó en la cuenca del río Andalién $\left(36^{\circ} 45^{\prime} \mathrm{S}, 72^{\circ} 50^{\prime} \mathrm{W}\right)$ en el Estero Nonguén (36ำ 49' a 36ำ $54^{\prime} \mathrm{S}, 72^{\circ} 57^{\prime}$ a $73^{\circ} 01^{\prime} \mathrm{W}$ ) de la Región del Biobío, Chile (ver figura 1). Este es un sistema fluvial de orden 3 (Habit et al., 2003), de $15 \mathrm{~km}$ de longitud y $44 \mathrm{~km}^{2}$ de superficie de cuenca hidrográfica (Jaque 1995), con una baja profundidad media $(<50 \mathrm{~cm})$. Posee un alto valor de conservación de su biodiversidad (Ortiz-Sandoval et al., 2009) en el que habitan 12 especies de peces, de las cuales 9 son nativas y 3 introducidas (Habit et al., 2003; Ruiz y Marchant, 2004). Actualmente, se encuentra altamente afectado por el encauzamiento de su rivera. El tramo analizado correspondió a la zona baja potamal del estero, es decir, un área de baja velocidad de corriente $\left(<0,5 \mathrm{~ms}^{-1}\right)$, substrato de arena, de mayor conductividad, presencia de macrófitas (Habit et al., 2003) y de alta biodiversidad (Ortiz-Sandoval et al., 2009).

\section{Evaluación de la Calidad del Sistema Ribereño}

Se utilizó el nivel de encauzamiento (END) como indicador para evaluar la calidad del tramo intervenido (ver ec. 1) que corresponde a la proporción entre el tramo de río canalizado y la longitud total del tramo evaluado, denominado longitud de masa de agua (Agència Catalana de l'aigua, 2006): 
$\mathrm{END}=\frac{\sum\left(\text { Longitud de encauzamiento }(\mathrm{m})^{*} \text { coeficiente }\right)}{\text { Longitud de masa de agua }(\mathrm{m})}$

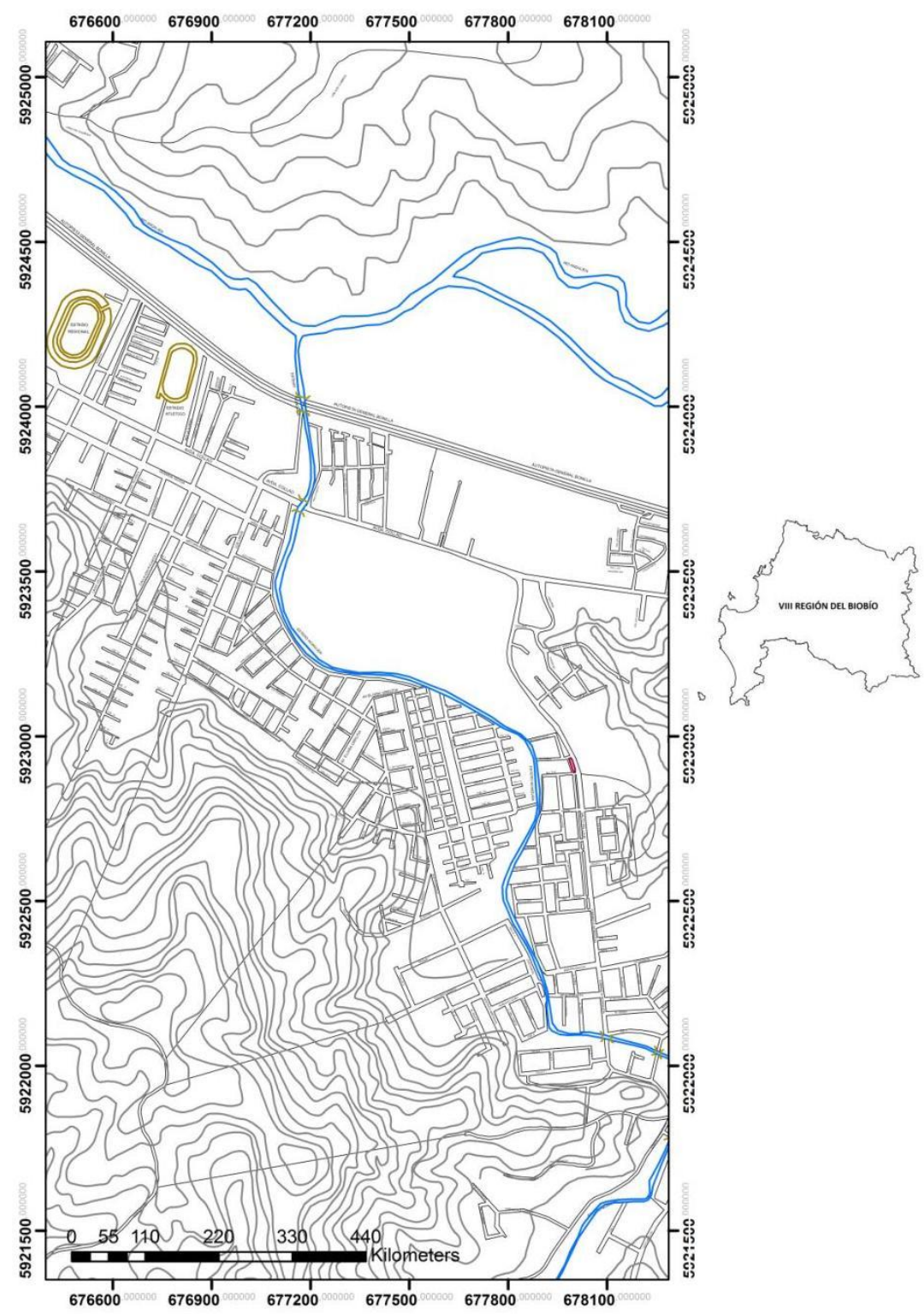

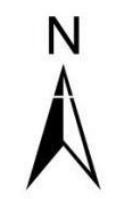

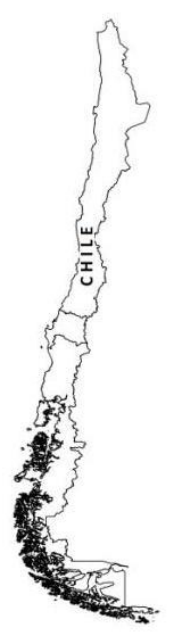

Fig.1: Área de estudio del Estero Nonguén, VIII Región del Biobío, Chile.

Se debe identificar el tipo de encauzamiento el cual puede ser de tipo mota, escollera o gavión, muro o muro en $U$ también denominado cauce hormigado, cuyo coeficiente de ponderación es igual a 0,2, 0,5, 0,8 y 1 respectivamente. En el caso que la obra afecte a una o ambas orillas se considera su efecto una o dos veces la longitud del encauzamiento, respectivamente (Agència Catalana de l'aigua, 2006). El nivel de calidad según el encauzamiento, será calificado según el valor del indicador END siendo de calidad muy bueno $(E N D<0,1)$, bueno $(0,1 \leq E N D<0,2)$, moderado $(0,2 \leq E N D<0,3)$ deficiente $(0,3 \leq E N D<0,4)$ y malo $(0,4 \leq E N D)$ (Agència Catalana de l'aigua, 2006).

\section{Evaluación del Índice de Conectividad Fluvial (ICF)}

De manera de identificar los puntos de conectividad crítica en el tramo del río, se evaluó el índice de conectividad fluvial (ICF) (Agència Catalana de l'aigua, 2006). Este índice considera las características de los peces que habitan en el sistema (Solá et al., 2011). Para ello se identificaron los sitios donde existían barreras para el movimiento de los peces, y se evaluaron sus dimensiones (Figura. 2).

Las especies nativas presentes en el Estero Nonguén fueron agrupadas según las características descritas en la metodología de la Agència Catalana de l'aigua (2006) constituido en seis tipos de grupos (Tabla 1) e información biológica conocida de las especies (Ortiz et al., 2009; Habit et al., 2003; Sobenes et al., 2012) 
clasificando a las especies nativas del Estero Nonguén según grupo (Tabla 2). Luego, con las características físicas de la barrera detectada, se identificaron los grupos de peces que sortearían el obstáculo (ver Tabla 3). Con ello, se consideró la valoración final de la barrera acorde a la Tabla 4.

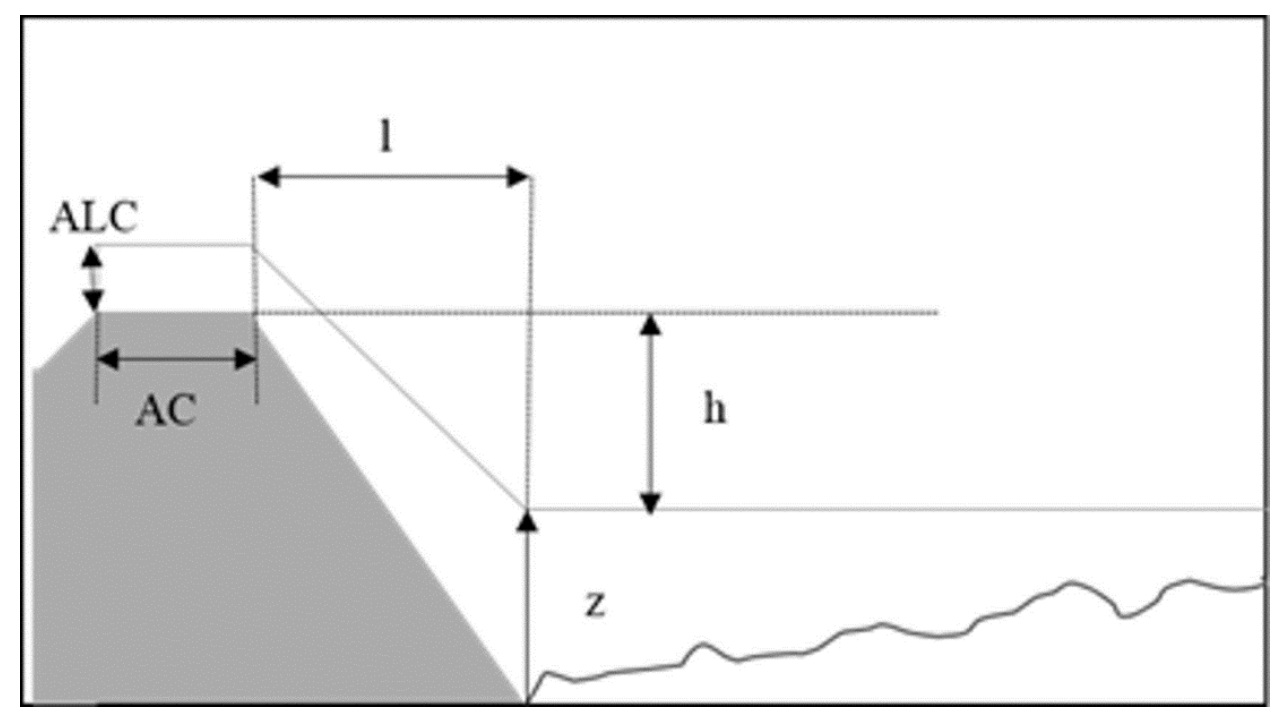

Fig. 2: Mediciones de la barrera (ALC: altura lámina de coronación; AC: ancho de coronación; I: longitud; h: altura y z: profundidad). (Adaptado de Agència Catalana de l'aigua, 2006).

Tabla 1: Características de los peces según grupo. (Adaptada de Agència Catalana de l'aigua, 2006)

\begin{tabular}{cl}
\hline Grupo & \multicolumn{1}{c}{ Característica } \\
\hline 1 & $\begin{array}{l}\text { Especies diádromas litorales de corto recorrido fluvial con baja capacidad para superar } \\
\text { obstáculos. }\end{array}$ \\
2 & $\begin{array}{l}\text { Especies catádromas de largo recorrido, sin capacidad de salto pero con elevada } \\
\text { capacidad para superar obstáculos. }\end{array}$ \\
3 & $\begin{array}{l}\text { Especies migratorias intrafluviales de corto recorrido, con capacidad para superar } \\
\text { obstáculos baja o moderada. Ciprínidos. }\end{array}$ \\
$3 a$ & Especies grandes, con capacidad moderada para superar obstáculos. \\
$3 b$ & Especies pequeñas, con muy poca capacidad para superar obstáculos. \\
4 & Especies migratorias intrafluviales con elevada capacidad natatoria y de salto.
\end{tabular}

Tabla 2: Clasificación de especies de peces presentes en el Estero Nonguén

\begin{tabular}{llc}
\hline \multicolumn{1}{c}{ Especie } & Tipo & Grupo \\
\hline Galaxias maculatus (Jenyns, 1842) & Nativa & 1 \\
Geotria australis (Gray, 1851) & Nativa & 1 \\
Percilia irwini (Jenyns, 1842) & Nativa & $3 b$ \\
Trichomycterus areolatus (Valenciennes, 1846) & Nativa & $3 b$ \\
Bullockia maldonadoi (Eigenmann, 1920) & Nativa & $3 b$ \\
Cheirodon galusdae Eigenmann, 1928 & Nativa & $3 b$ \\
Nematogenys inermis (Guichenot, 1848) & Nativa & $3 b$ \\
Percichthys trucha (Valenciennes, 1833) & Nativa & $3 b$ \\
Basilichthys australis Eigenmann, 1928 & Nativa & $3 b$ \\
Gambusia affinis (Baird y Girard, 1853) & Invasora & $3 b$ \\
Oncorhynchus mykiss (Walbaum, 1792) & Invasora & 4 \\
Salmo trutta Linnaeus, 1758 & Invasora & 4 \\
\hline
\end{tabular}


Tabla 3: Grupos de peces que sortean la barrera según sus dimensiones. Adaptado Agència Catalana de l'aigua (2006)

\begin{tabular}{lllccccccc}
\hline \multicolumn{1}{c}{ Verticalidad } & Altura $(\mathrm{cm})$ & $\begin{array}{c}\text { Profundidad } \\
(\mathrm{cm})\end{array}$ & $\begin{array}{c}\text { Ancho de } \\
\text { coronación } \\
(\mathrm{cm})\end{array}$ & $\begin{array}{c}\text { Altura de } \\
\text { lámina } \\
\text { coronación }(\mathrm{cm})\end{array}$ & \multicolumn{3}{c}{ Grupos que pasan } & \\
\hline Indiferente & $\mathrm{h} \leq 10$ & $\mathrm{z} \geq 15$ & $\mathrm{AC} \leq 50$ & - & $\mathrm{G} 1$ & $\mathrm{G} 2$ & $\mathrm{G} 3 \mathrm{a}$ & $\mathrm{G} 3 \mathrm{~b}$ & $\mathrm{G} 4$ \\
Indiferente & $\mathrm{h} \leq 10$ & $\mathrm{z} \geq 15$ & $\mathrm{AC}>50$ & $\mathrm{ALC} \geq 15$ & $\mathrm{G} 1$ & $\mathrm{G} 2$ & $\mathrm{G} 3 \mathrm{a}$ & $\mathrm{G} 3 \mathrm{~b}$ & $\mathrm{G} 4$ \\
Indiferente & $\mathrm{h} \leq 20$ & $\mathrm{z} \geq 30$ & $\mathrm{AC} \leq 50$ & - & $\mathrm{G} 1$ & $\mathrm{G} 2$ & $\mathrm{G} 3 \mathrm{a}$ & $\mathrm{G} 3 \mathrm{~b}$ & $\mathrm{G} 4$ \\
Indiferente & $\mathrm{h} \leq 20$ & $\mathrm{z} \geq 30$ & $\mathrm{AC}>50$ & $\mathrm{ALC} \geq 15$ & $\mathrm{G} 1$ & $\mathrm{G} 2$ & $\mathrm{G} 3 \mathrm{a}$ & $\mathrm{G} 3 \mathrm{~b}$ & $\mathrm{G} 4$ \\
Indiferente & $\mathrm{h} \leq 30$ & $\mathrm{z} \geq 40$ & - & - & & $\mathrm{G} 2$ & $\mathrm{G} 3 \mathrm{a}$ & & $\mathrm{G} 4$ \\
Vertical & $\mathrm{h} \leq 40$ & $\mathrm{z} \geq 60$ & - & - & $\mathrm{G} 2$ & $\mathrm{G} 3 \mathrm{a}$ & & $\mathrm{G} 4$ \\
Indiferente & $\mathrm{h} \leq 60$ & $\mathrm{z} \geq 70$ & - & - & $\mathrm{G} 2$ & & & $\mathrm{G} 4$ \\
Vertical & $\mathrm{h} \leq 75$ & $\mathrm{z} \geq 90$ & - & - & $\mathrm{G} 2$ & & & $\mathrm{G} 4$ \\
Indiferente & Indiferente & Indiferente & $\mathrm{AC}>50$ & $0<\mathrm{ALC}<15$ & $\mathrm{G} 2$ & & & \\
\hline
\end{tabular}

Tabla 4: Valoración de la barrera para el movimiento de los peces. Fuente: Agència Catalana de l'aigua, 2006

\begin{tabular}{ll}
\hline \multicolumn{1}{c}{ Condición de paso de los peces } & \multicolumn{1}{c}{ Valoración } \\
\hline Si pasan todos los grupos presentes & $\begin{array}{l}\text { Barrera franqueable para las especies de los } \\
\text { grupos presentes } \\
\text { Si algún grupo presente no pasa la barrera }\end{array}$ \\
$\begin{array}{l}\text { Barrera infranqueable para alguno de los } \\
\text { grupos presentes }\end{array}$ \\
$\begin{array}{l}\text { continuán grupo no pasa la barrera o bien no circula agua de forma } \\
\text { vados agujereados) }\end{array}$ & $\begin{array}{l}\text { Barrera infranqueable para todos los grupos } \\
\text { presentes }\end{array}$ \\
\hline
\end{tabular}

Según las características de los obstáculos, finalmente se evaluó el nivel de calidad de la conectividad del sistema ribereño en una escala cualitativa, siendo el nivel de calidad muy bueno (sin obstáculo), bueno (obstáculo franqueable), deficiente (obstáculo infranqueable para algunos grupos de peces) y malo (obstáculo infranqueable para todos los grupos de peces) (Agència Catalana de l'aigua, 2006).

\section{Selección del Paso de Peces}

Para proponer una alternativa de paso de peces, se utilizó el método de decisión multicriterio ponderación lineal o scoring (Berumen y Llamazares, 2007). El método construye una función de valor para cada alternativa permitiendo elegir dentro de varias alternativas, maximizando la preferencia según varios criterios (Anderson et al., 2011; Tavella et al., 2014). Para ello, se siguieron cinco pasos: i) Definición de meta u objetivo global que se desea alcanzar; ii) Identificación de criterios (puntos de vista importantes para resolver el problema o alcanzar el objetivo); iii) Identificación de alternativas mediante las cuales se podría alcanzar el objetivo global; iv) Construcción de un diagrama de jerarquías; v) Asignar una ponderación para cada uno de los criterios.

Hurtado y Bruno (2005) plantean que la asignación de pesos a los criterios por el decidor puede ser manipulable, lo cual quita validez al método. Es por esto que, para obtener los pesos preferenciales para cada uno de los criterios, sólo se consultó al decidor que ordenara los criterios por orden decreciente de importancia, para poder aplicar la siguiente expresión:

$$
W_{i}=\frac{1 / \sum_{j=1}^{n} r_{i}}{\sum_{j=1}^{n} 1 / r_{i}}
$$

donde $r_{i}$ representa la posición que ocupa el i-ésimo criterio en la clasificación establecida i= 1,..,n (Martínez, 1983).

Posteriormente, se evaluó en cuánto satisface cada alternativa a nivel de cada uno de los criterios. Para ello, se calculó el peso global (S) de cada una de las alternativas (ver ec. 3).

$$
S_{j}=\sum_{i} W_{i} p_{i j}
$$

donde $w_{i}$ : es la ponderación de cada criterio i; $p_{i j}$ : ponderación de la alternativa j en función del criterio i y $S_{j}$ : el peso global de la alternativa $j$. 
Luego, las alternativas evaluadas se ordenaron de mayor a menor y aquella con el puntaje más alto correspondió a la alternativa a proponer. El rating de satisfacción para cada alternativa se clasificó acorde al puntaje obtenido, siendo clasificado como muy malo (puntaje $\leq 20)$, malo $(20<$ puntaje $\leq 40)$, regular $(40<$ puntaje $\leq 60)$, bueno $(60$ puntaje $\leq 80)$ y muy bueno $(80<$ puntaje $\leq 100)$. Se definieron tres niveles, donde el nivel 1 corresponde al objetivo seleccionar un dispositivo de paso de peces; el nivel 2 son los criterios considerados que fueron: el costo del paso de peces, que fuera de tránsito simple y que no altere negativamente al ambiente; y el nivel 3 fueron las alternativas de paso de peces evaluadas: pasos rústicos, rampas de piedra y río artificial (Confederación Hidrográfica del Ebro, 2009).

\section{RESULTADOS Y DISCUSIÓN}

En el tramo analizado de $2.275 \mathrm{~m}$ se identificaron nueve tipos de obras (ver Tabla 5). Según el nivel de encauzamiento (END), a este tramo del sistema ribereño se clasificó en calidad moderada (Tabla 5).

Tabla 5: Calificación del sistema fluvial Estero Nonguén

\begin{tabular}{|c|c|c|c|c|c|}
\hline \multirow{2}{*}{ Tipo de obra } & \multirow{2}{*}{ Coeficiente (c) } & \multirow{2}{*}{$\begin{array}{c}\text { Longitud } \\
\text { encauzamiento }(m)\end{array}$} & \multicolumn{2}{|c|}{$\begin{array}{l}\text { Presencia de márgenes } \\
(p m)\end{array}$} & \multirow{2}{*}{$c^{*} l^{*} p m$} \\
\hline & & & Un margen & $\begin{array}{c}\text { Ambos } \\
\text { márgenes }\end{array}$ & \\
\hline Escollera & 0,5 & 10 & & $x$ & 10 \\
\hline $\begin{array}{l}\text { Escollera (resto margen } \\
\text { derecho) }\end{array}$ & 0,5 & 2,8 & $x$ & & 1,4 \\
\hline Gavión & 0,5 & 132,2 & & $\mathrm{x}$ & 132,2 \\
\hline Gavión (resto margen izquierdo) & 0,5 & 8,3 & $\mathrm{x}$ & & 4,15 \\
\hline Muro en U 1 (D) & 1 & 32 & $x$ & & 32 \\
\hline Muro 2 (D) & 0,8 & 33,1 & $x$ & & 26,48 \\
\hline Muro $3(D)$ & 0,8 & 71,5 & $\mathrm{x}$ & & 57,2 \\
\hline Escollera 2 (D) & 0,5 & 150,1 & $\mathrm{x}$ & & 75,05 \\
\hline Muro 1 (I) & 0,8 & 282 & $x$ & & 225,6 \\
\hline Sumatoria & & & & & 564,08 \\
\hline Longitud de masa de agua $(\mathrm{m})$ & & & & & 2.275 \\
\hline END & & & & & 0,25 \\
\hline
\end{tabular}

\section{Índice de Conectividad Fluvial}

En el tramo estudiado, se identificó una barrera artificial correspondiente a una tubería transversal con una estructura tipo azud (dimensiones $h=29,8 \mathrm{~cm}, \mathrm{l}=25 \mathrm{~cm}, \mathrm{z}=40,5 \mathrm{~cm}, A C=30 \mathrm{~cm}$ y $\mathrm{ALC}=30,3 \mathrm{~cm}$ ). La barrera es infranqueable por las especies del grupo 1 y $3 b$ (Tabla 2 y 3 ), por lo que se clasifica al tramo estudiado con un ICF deficiente (Tabla 4).

\section{Propuesta de la alternativa de paso para peces}

Los criterios fueron ordenados de manera decreciente en importancia y pesos, siendo: primero que permitiera un tránsito sencillo $\left(\mathrm{W}_{1}=0,54\right)$, segundo de bajo costo $\left(\mathrm{W}_{2}=0,27\right)$ y por último que no afectara al ambiente $\left(W_{3}=0,18\right)$. La evaluación de las alternativas, según los criterios y pesos corres-pondientes, determinó que la alterantiva seleccionada es el paso de peces rampas de piedra (Tabla 6).

Tabla 6: Evaluación para la selección del paso de peces del tramo estudiado en el Estero Nonguén.

\begin{tabular}{lcccc}
\hline \multirow{2}{*}{ Alternativas } & \multicolumn{5}{c}{ Criterios } \\
\cline { 2 - 5 } & $\begin{array}{c}\text { Tránsito sencillo } \\
\left(\mathrm{W}_{1}=0,54\right)\end{array}$ & $\begin{array}{c}\text { Costo de ejecución } \\
\left(\mathrm{W}_{2}=0,27\right)\end{array}$ & $\begin{array}{c}\text { Acorde al ambiente } \\
\left(\mathrm{W}_{3}=0,18\right)\end{array}$ & $\begin{array}{c}\text { Puntaje } \\
\text { total }\end{array}$ \\
\hline Pasos rústicos & 60 & 90 & 50 & 65,7 \\
Rampas de piedra & 80 & 80 & 60 & 75,6 \\
Río artificial & 90 & 40 & 80 & 73,8 \\
\hline
\end{tabular}

De esta manera, en este estudio se encontró que la calidad evaluada del tramo del sistema ribereño Estero Nonguén, mediante el nivel de encauzamiento END y el índice de conectividad fluvial ICF, es de calidad 
moderada y de conectividad deficiente, producto de la intervención con obras civiles para la canalización de la ribera. Esto coincide con lo encontrado por Correa-Araneda y Salazar (2014) quienes describen que en la parte baja del Estero Nonguén baja su calidad debido a la intensidad de la actividad humana. Así mismo, Palma et al. (2009) describen que a medida que se alejan de la parte alta del cauce existe una tendencia a la degradación de la ribera, disminuyendo considerablemente la cubierta vegetal y la zona de inundación. Estos indicadores de evaluación aplicados muestran que son fiables, considerando además que han sido amplia y exitosamente aplicados en la evaluación de sistemas ribereños del mundo (Ramírez, 2015; Solà et al., 2011).

La barrera identificada en el tramo analizado del Estero Nonguén, correspondiente a una tubería transversal, determina la conectividad deficiente del sistema ribereño, lo que significa que sólo una o varias especies de los potenciales grupos de especies que existen en el lugar pueden pasar y en determinadas condiciones hidrológicas (Solà et al., 2011). Por lo tanto, es imperante una solución a este impedimento de desplazamiento de la fauna íctica siendo posible la eliminación del obstáculo o la incorporación de un dispositivo de paso (WWF, 2015). Aunque la solución más efectiva es la eliminación del obstáculo, se requiere mantener estructuras que permita el cumplimiento de dos objetivos, una obra civil que cumple una función de servicios a una población aledaña a la ribera, y de mantener la conectividad del cauce. A pesar de que no existen guías para proponer diseños de estructuras, considerando los múltiples factores a considerar, una de las alternativas de mayor preferencia son los pasos naturalizados como la rampa de piedras (Bunt et al., 2012). Los autores Sanz-Ronda et al. (2011) plantean que este tipo de paso se adapta mejor a obstáculos de pequeña y mediana altura. En este estudio al realizar la evaluación del dispositivo, se encuentra como alternativa la rampa de piedra, un paso de peces naturalizado, de bajo costo, transito sencillo y acorde al ambiente ribereño.

Aunque los estudios de dispositivos de pasos se encuentran en desarrollo a nivel mundial, diversos autores abordan su desarrollo destacando la necesidad del enfoque interdisciplinar (Beach, 1984; Sanz-Ronda et al., 2013; FAO-DVWK, 2002; Pena et al., 2006, Schilt, 2007; Katopodis, 2013). No obstante, este es un tema de bajo desarrollo en Chile, a pesar de la necesidad inminente de conservar la ictiofauna nativa chilena y del manejo integral de los ríos chilenos y la calidad físico química de ellos (Rivera et al., 2004). Lo anterior, exige realizar mayor investigación sobre velocidades de natación en peces, uso de hábitats y comportamiento de los peces frente a distintos tipos de condiciones de flujos y ambientales (Williams et al., 2012) así como frente a diferentes diseños de estructuras para el paso de peces (Doehring et al., 2011). Todo lo anterior, involucra un desafío político, económico y ecológico (Katopodis y Williams, 2012), que permita la conservación de la biodiversidad en aguas continentales.

\section{CONCLUSIONES}

Según el objetivo del presente estudio, la calidad de un tramo del estero Nonguén fue calificada de calidad moderada y de una conectividad deficiente. Esto implica que existirían grupos de peces nativos que no se pueden desplazar aguas arriba de la barrera ni aguas debajo de la barrera.

Para restablecer la conectividad se propone como solución la instalación de un dispositivo de rampa de piedras, correspondiente a un sistema de bajo costo, tránsito sencillo y acorde al ambiente ribereño.

La metodología propuesta, fue aplicable modificando algunos criterios acordes al conocimiento de expertos y literatura nacional. Este análisis puede ser replicado a ríos y esteros nacionales ubicados en zonas urbanas e intervenidas, de manera de implementar acciones de gestión para mitigar los impactos generados por las obras civiles sobre cursos de agua y mantener la biodiversidad de los ríos chilenos.

\section{AGRADECIMIENTOS}

Se agradece a la Dirección de Investigación de la Universidad Católica de La Santísima Concepción por su financiamiento (Proyecto DIN 10/2014).

\section{REFERENCIAS}

Agència Catalana de l'Aigua. HIDRI: Protocolo para la valoración de la calidad hidromorfológica de los ríos. Barcino Solucions Gràfiques, 1- 131, Barcelona, España (2006)

Anderson, D. R.; D. Sweeney; T.A. Williams; J.D. Camm y K. Martin. Métodos cuantitativos para los negocios. $11^{\text {a }}$ Ed. Learning Editores S.A., 840, Santa Fé, México (2011)

Beach, M. Fish pass design-criteria for the design and approval of fish passes and other structures to facilitate the passage of migratory fish in rivers. Ministry of Agriculture, Fisheries and Food, Directorate of Fisheries Research, Lowestoft (1984)

Berumen, S.A. y F. Llamazares. La utilidad de los métodos de decisión multicriterio (como el AHP) en un entorno de competitividad creciente. Cuadernos de Administración, 20(34), 65-87 (2007) 
Brooker, M. The ecological effects of channelization. The Geographical Journal, 151(1), 63-69 (1985)

Bunt, C.M.; T. Castro-Santos y A. Haro. Performance of fish pass structures at upstream barriers to migration. River Research and Applications, 28, 457-478 (2012)

Confederación Hidrográfica del Ebro, Pasos de peces para permeabilizar estructuras transversales en la cuenca del Ebro (en línea: http://195.55.247.234/webcalidad/estudios/2009_Protocolo_Permeabilizacion_Obstaculos.pdf, acceso: 15 noviembre 2015), Ministerio de Medio Ambiente y Medio Rural y Marino, España (2009)

Correa-Araneda, F. y C. Salazar. Caracterización físicoquímica del agua del estero Nonguén y su confluencia con el río Andalién, Región del Biobío. Variación en relación a los distintos usos de suelo en su cuenca. Sustainability. Agri., Food and Environmental Research, 2(2), 33-46 (2014)

Doehring, K.; R. Young y A. McIntosh. Factors affecting juvenile galaxiid fish passage at culverts. Marine and Freshwater Research, 62, 38-45 (2011)

Elvira, B.; G.G. Nicola; y otros cuatro autores. Impacto de las obras hidráulicas en la ictiofauna. Dispositivos de paso para peces en las presas de España (en línea: https://goo.gl/MmsSwV acceso: 01 noviembre 2016), Organismo Autónomo Parques Nacionales, Madrid, España (1998)

Food and Agriculture Organization of the United Nations (FAO)/Deutcher Verband fur Wasserwirtschaft und Kulturbau (DVWK) Fish passes- design, dimensions and monitoring (en línea: https://goo.gl/Kp9rVa), Roma, Italia (2002)

Gardner, E. Massive Chilean dams approved. Nature News, 297 (2011)

Goodwin, P.; K. Jorde; C. Meier y O. Parra. Minimizing environmental impacts of hydropower development: transfering lessons from the past projects to a proposed strategy for Chile. Journal of Hydroinformatics, 8, 253-270 (2006)

Habit, E. y P. Victoriano. Composición, origen y valor de conservación de la Ictiofauna del Río San Pedro (Cuenca del Río Valdivia, Chile). Gayana, 76, 10-23 (2012)

Habit, E.; P. Victoriano y A. Rodríguez-Ruiz. Variaciones espacio-temporales del ensamble de peces de un sistema fluvial de bajo orden del centro-sur de Chile. Revista Chilena de Historia Natural, 76, 3-14 (2003)

Hurtado, G. El Proceso de análisis jerárquico (AHP) como herramienta para la toma de decisiones en la selección de proveedores: aplicación en la selección del proveedor para la Empresa Gráfica Comercial MyE S.R.L. Tesis de Licenciado en Investigación Operativa. Facultad de Ciencias Matemáticas, Universidad Nacional Mayor de San Marcos, Perú (2005)

Jackson, D.; P. Peres-Neto y J. Olden. What controls who is where in freshwater fish communities the roles of biotic, abiotic, and spatial factors. Canadian Journal of Fisheries and Aquatic Sciences, 58(1), 157- 170 (2001)

Jaque, E. Análisis integrado de los sistemas naturales de la cuenca del río Andalién: bases para la planificación ecológica del territorio de la cuenca. Tesis Doctoral, Centro EULA, Universidad de Concepción, Concepción, Chile (1995)

Katopodis, C. y J. Williams. The development of fish passage research in a historical context. Ecological Engineering, 48 , 8-18 (2012)

Katopodis, C. Developing a toolkit for fish passage, ecological flow management and fish habitat works. Journal of Hydraulic Research, 43 (5), 451-467 (2013)

Kemp, P. Bridging the gap between fish behavior, performance and hydrodynamics: an ecohydraulics approach to fish passage research. River Research and Applications, 28, 403-406 (2012)

Mallen-Cooper, M. y D. Brand. Non-salmonids in a salmonid fishway: what do 50 years of data tell us about past and future fish passage? Fisheries Management and Ecology, 14(5), 319-332 (2007)

Martínez, E. Evaluación y decisión multicriterio: reflexiones y experiencias. Mauricio Escudey Ed. 1-222. Universidad de Santiago, Santiago, Chile (1998)

Martínez, E. Hidráulica fluvial: Principios y práctica. Bellisco Eds., Madrid, España (2001)

Mims, M. y J. Olden. Life history theory predicts fish assemblage response to hydrologic regimes. Ecology, 93, 35-45 (2012)

Morita, K y S. Yamamoto. Effects of Habitat Fragmentation by Damming on the Persistence of Stream-Dwelling Charr Populations. Conservation Biology, 16(5), 1318-1323 (2002)

Morita, K. y A. Yokota. Population viability of stream-resident salmonids after habitat fragmentation: a case study with white-spotted charr (Salvelinus leucomaenis) by an individual based model. Ecological Modelling, 155, 85-94 (2002)

Morita, K. y A. Yokoto. Population viability of stream-resident salmonids after habitat fragmentation: a case study with white-spotted charr (Salvelinus leucomaenis) by an individual based model. Ecological Modelling, 155(1), 85-94 (2002)

Morita, K. y S. Yamamoto. Effects of habitat fragmentation by damming on the persistence of stream-dwelling charr populations. Conservation Biology, 16, 1318 - 1323 (2002)

Ortiz-Sandoval J.J.; N. Ortiz; R. Cifuentes; J. Gonzales y E. Habit. Respuesta de la comunidad de peces al dragado de ríos costeros de la región del BioBío (Chile). Gayana, 73(1), 64-75 (2009)

Palma, A. R. Figueroa y V. Ruiz. Evaluación de rivera y hábitat fluvial a través de los índices QBR e IHF. Gayana, 73(1), 57-63 (2009) 
Pena, L.; J. Puertas; T. Teijeiro y E. Peña, Dispositivos de remonte para peces: escalas de hendiduras verticales. Ingeniería del Agua, 13(2), 113-128 (2006)

Piedra, P.; E. Habit y otros siete autores. Patrones de desplazamiento de peces nativos en el Río San Pedro (cuenca del Río Valdivia, Chile). Gayana 76, 59-70 (2012)

Pompeu, P.; A. Agostinho y F. Pelicice. Existing and future challenges: the concept of succecsful fish passage in South America. River Research Application, 28, 504-512 (2012)

Ramírez, J.L. Propuesta metodológica para la valoración ambiental de corrientes hídricas desde la perspectiva de la restauración fluvial Caso de estudio Quebrada Olivares-Minitas Manizales (Caldas). Tesis de Magister en Medio Ambiente y Desarrollo, Facultad Ingeniería y Arquitectura, Universidad Nacional de Colombia, Colombia, 286 p. (2015)

Rivera, N.R; F. Encina; A. Muñoz-Pedreros y P. Mejías. La Calidad de las Aguas en los Ríos Cautín e Imperaila, IX Región - Chile, Información Tecnológica, 15 (5), 89-101 (2004)

Ruiz, V. y M. Marchant. Ictiofauna de aguas continentales chilenas. 1-356. Universidad de Concepción, Concepción, Chile (2004)

Sanz-Ronda, F.; A. García y J. Fuentes. Evaluación hidráulica de un paso naturalizado (río artificial) en el Marín (Río Tormes, Salamanca). Actas del I Congreso Ibérico de restauración fluvial RESTAURARíOS, 435-442, León, España 18 20 octubre (2011)

Sanz-Ronda, F.; F. Bravo; y otros cinco autores. Pasos para peces: escalas y otros dispositivos de paso. Notas Técnicas Del CIREF, 7, 1-17 (2013)

Schilt, C., Developing fish passage and protection at hydropower dams. Applied Animal Behaviour Science 104(3), 295$325(2007)$

Sobenes, C.; A. García; E. Habit, y O. Link, Mantención de peces nativos dulceacuícolas en Chile en cautiverio: un aporte a su conservación ex situ. Boletín de Biodiversidad de Chile, 7, 27-41 (2012)

Solà, C. M. Ordeix, Qy otros seis autores, Longitudinal connectivity in hydromorphological quality assessments of rivers. The ICF index: A river connectivity index and its application to Catalan rivers. Limnetica, 30(2), 273-292 (2011)

Swales, $\mathrm{S}$. The use of instream habitat at improvement methodology in mitigation the adverse effects of river regulation on fisheries. 185-208, En J.A. Gore y G. E. Petts, eds. Alternatives in regulated river management. Chemcial Rubber Company Press, Boca Ratón, Florida, Estados Unidos (1989)

Tavella, M.; A. Miropolsky y R. Manera. Estudio Comparativo de Métodos Multicriterio para el análisis de la Localización Sustentable de Parques Industriales Regionales. Revista de la Facultad de Ciencias Exactas, Físicas y Naturales, 1(1), 41-48 (2014)

Trapote, B. Estudio teórico de pasos de peces y desarrollo de una metodología de evaluación de su eficacia. 110 p. Tesis de Ingeniero Civil. Universitat Politècnica de Catalunya, España (2009)

Vannote, G.; K. Cummins; J. Sedell, y C. Cushing. The river continuum concept. Canadian Journal of Fisheries and Aquatic Science, 37, 130-137 (1980)

Vila, I. y E. Habit. Current situation of the fish fauna in the Mediterranean region of Andean river systems in Chile. FISHMED Fishes in Mediterranean Environments, 2 (2015)

Vila, I.; L. Fuentes y M. Contreras. Peces límnicos de Chile. Boletín del Museo Nacional de Historia Natural. Chile, 48, 6175 (1999)

Vince, G. Dams for Patagonia. Science, 329, 382-385 (2010)

Washington Department of Fish and Wildlife. Fish Passage and Surface Water Diversion Screening Assessment and Prioritization Manual. 240 p. Washington Department of Fish and Wildlife. Olympia, Washington. United States (2009)

Williams, J., S. Smith, y otros cinco autores. Effects of the federal Columbia River power system on salmon populations. NOAA Technical Memorandum, NMFS-NWFSC (2005)

Williams, J.; G. Armstrong; C. Katopodis; M. Larinier y F. Travade. Thinking like a fish: a key ingredient for development of effective fish passage facilities at river obstruction. River Research Application, 28, 407-417 (2012)

WWF, Recomendaciones y buenas prácticas de gestión en espacios fluviales. Para gestores, técnicos de organismos de cuenca, administraciones y entidades que gestionan espacios fluviales (en línea: https://goo.gl/LmKvEJ, acceso 15 de octubre 2015), Madrid, España (2015) 
